\title{
Erratum: rf system models for the CERN Large Hadron \\ Collider with application to longitudinal dynamics \\ [Phys. Rev. ST Accel. Beams 13, 102801 (2010)]
}

T. Mastorides, C. Rivetta, J. D. Fox, D. Van Winkle, and P. Baudrenghien (Received 31 January 2011; published 14 February 2011)

We have identified an omission in our recent paper. A negative sign is missing from the exponent in Eq. (14). The corrected equation is

$$
\sigma_{l}+j \Delta \omega_{l}=\frac{\eta q I_{o}}{2 \beta^{2} \omega_{s} E_{o} T_{o}} \sum_{p=-\infty}^{\infty} Z(\omega) \omega e^{-\sigma_{\tau}^{2} \omega^{2}} .
$$

The results and conclusions of the article are not effected. 